\title{
A Precise Experimental Elucidation of the Apparent Vertical Effects of Spinning Gyroscopes ${ }^{\dagger}$
}

\author{
Susumu IKEDA*, Takuma DoI*, \\ Keizaburo UCHIKAWA* and Yoshiharu KURITA*
}

\begin{abstract}
The existence of intrinsic vertical effects of spinning gyroscopes was examined systematically from the viewpoint of precise measurement, using three types of gyroscopes with largely different characteristics. The most precise weighing techniques, such as a reference tare system and various countermeasures, were applied. Slight and symmetrial mass changes observed in the experiment were explained as the parasitic effect of the balance due to the torque of the spinning gyroscopes, utilizing the attenuation constant change under atmospheric conditions. Consequently, the intrinsic vertical effects were found to be virtually zero in this experiment, and even if existent, were estimated to be neither greater than $+0.7 \mathrm{mg}$ nor less than $-0.5 \mathrm{mg}$ at a $99 \%$ confidence level for the angular momentum of $0.936 \mathrm{~kg} \cdot \mathrm{m}^{2} / \mathrm{s}$.
\end{abstract}

Key Words: weight reduction, spinning gyroscope, rotational speed, reference tare system, parasitic effect of balance

\section{Introduction}

Anomalous weight reduction in spinning gyroscopes was reported by Hayasaka and Takeuchi ${ }^{1}$. They concluded, on the basis of their experiment, that for right (spin vector down) spin about a vertical axis, the masses of the gyroscopes seemed to decrease linearly according to the masses and the rotational speeds of the rotor, while for left spin, they did not. Subsequently, four pieces of counterevidence were reported for such a large and asymmetric effect ${ }^{2) \sim 5)}$.

The first letter, however, was the first that reported the vertical effect of a spinning gyroscope under vacuum. Therefore, we believe that we should examine such effects from the viewpoint of precise measurement, without assigning too much weight to the experimental formula proposed by the first authors. From this point of view, there are disagreements in the magnitude of the weight change appearing in these papers, including this one.

Since each author arguing against the weight reduc-

$\dagger$ 計測自動制御学会質量・力学部会講演会 $(1990 \cdot 11)$, 応用連合春季講演会 $(1991 \cdot 3)$ で発表

* National Research Laboratory of Metrology, 1-1-4, Umezono, Tsukuba, Ibaraki

(Received January 29, 1992)

(Revised September 3, 1992) tion effects used a different gyroscope, the general aspects of the magnitude of the observed effects with respect to spinning gyroscopes are difficult to discuss. Their weighing accuracies are sufficient for the discussion of their results against those of the first authors; however, they are insufficient for the discussion of whether the slight observed weight change in their experiments was intrinsic or their weighing accuracy was good enough. They discussed their results without separating the systematic errors of their weighing systems and the parasitic effects of their balances due to the torque of their spinning gyroscopes from their respective experimental results.

Therefore, to discuss the observed effect more systematically and accurately, we examined three types of gyroscopes with largely different characteristics in the mass and the running time of inertial rotation of the rotor (hereafter referred to as "the running time"), using our high weighing potential. Much time was spent in preparation and elaborate measurements.

The results were naturally negative for such effects as asymmmetric ones, those due to the direction of rotation, the rotational speed $\omega$ and the derivative $\omega^{\prime}$ of $\omega$ with respect to time. In the cases of gyroscopes 
with large angular momentum, slight and symmetrical weight changes were observed.

Whether the effect is intrinsic or not both under vacuum and at atmospheric pressure will be discussed in this paper.

\section{Gyroscopes and Measuring System}

\subsection{Gyroscopes}

First, gyroscopes with rotors that were large in size and mass were chosen : they are naturally preferable to gyroscopes with small rotors because larger

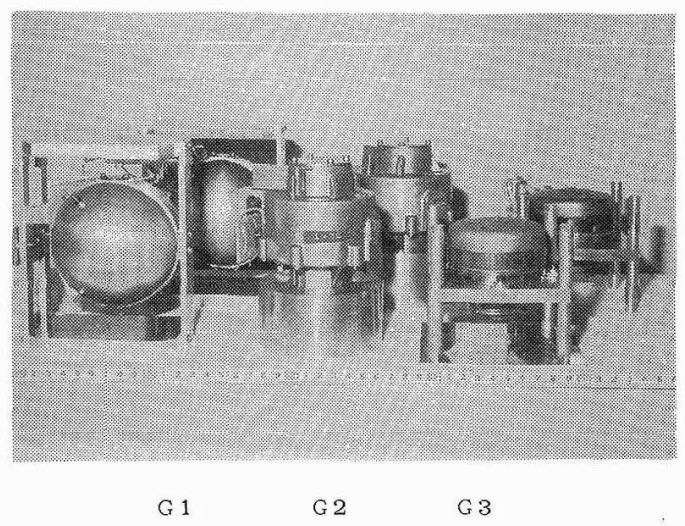

Photo. 1 Photograph of gyroscopes used. Each kind of gyroscopes is used in a pair, one of which is used as a sample and the other as a reference. effects, if existing, can be observed.

Next, gyroscopes with different characteristics of inertial rotation had to be selected; $\omega^{\prime}$ may be thought to cause such an effect instead of $\omega$ because $\omega^{\prime}$ decreases exponentially, as does $\omega$. Therefore, three types of commercially available gyroscopes with largely different characteristics in the mass of the rotor and the running time were selected; all types had electric motors for driving and each of them was used in a pair, as will be described in greater detail later. These photographs and their characteristics are shown in Photo. 1 and Table 1, respectively. In particular, it is noteworthy that gyroscopes with rotor mass of over $1 \mathrm{~kg}$ and running time of over 40 minutes were included because no one had experimented with such gyroscopes yet.

\subsection{Weighing System}

A highly precise tare system called the "differential substitution system" or "reference tare system" was adopted; it requires a different tare (hereafter referred to as the "RT") from the usual balancing tare, substituted for a sample on the same pan of a balance.

Photo. 2 shows the sample on the pan and the RT on the opposite weight stage of the turntable. The RT was composed of the same elements as the sample. The elements were as follows: (1) a vacuum container with a hermetic seal, having dimensions of

Table 1 Characteristics and specifications of gyroscopes used

\begin{tabular}{|c|c|c|c|c|c|c|c|c|c|}
\hline $\begin{array}{l}\text { Sym- } \\
\text { bol }\end{array}$ & $\begin{array}{c}\text { Rated rotati- } \\
\text { onal speed } \\
\omega_{r} \\
(\mathrm{rpm}) \\
\end{array}$ & $\begin{array}{c}\text { Rated angular } \\
\text { momentum at } \omega_{\mathrm{r}} \\
\mathrm{H} \\
\left(\mathrm{kg} \cdot \mathrm{m}^{2} / \mathrm{s}\right)\end{array}$ & $\begin{array}{c}\text { Mass of } \\
\text { rotor } \\
\mathrm{m} \\
(\mathrm{kg})\end{array}$ & \begin{tabular}{|c|} 
Effective \\
radius of \\
rotor $r_{e f}$ \\
$(\mathrm{~cm})$
\end{tabular} & \begin{tabular}{|c|} 
Expected \\
mass reduc- \\
tion $\Delta \mathrm{m}_{\mathrm{R}}{ }^{*}$ \\
$(\mathrm{mg})$
\end{tabular} & $\begin{array}{l}\text { Running time } \\
\text { of inertial } \\
\text { rotation } \tau^{* *} \\
\quad \text { (min) }\end{array}$ & $\begin{array}{l}\text { Driving } \\
\text { type of } \\
\text { rotor }\end{array}$ & $\begin{array}{l}\text { type of } \\
\text { motor }\end{array}$ & Remarks \\
\hline G1 & 11700 & 1.217 & 1.15 & 2.82 & -86 & $\begin{array}{l}\tau_{\mathrm{v}} \fallingdotseq 42 \\
\tau_{\mathrm{A}} \fallingdotseq 17\end{array}$ & \begin{tabular}{c|} 
inner \\
rotor
\end{tabular} & \begin{tabular}{|l|} 
synchro- \\
nous \\
motor
\end{tabular} & $\begin{array}{l}\text { GM-10 (GC }) \\
\text { TOKIMEC Co. } \\
\text { Ltd. }\end{array}$ \\
\hline $\mathrm{G} 2$ & 11700 & 1.217 & 1.08 & 2.91 & -81 & $\begin{array}{l}\tau_{\mathrm{V}} \fallingdotseq 13 \\
\tau_{\mathrm{A}} \fallingdotseq 11\end{array}$ & $\begin{array}{c}\text { outer } \\
\text { rotor }\end{array}$ & $\begin{array}{l}\text { synchro- } \\
\text { nous } \\
\text { motor }\end{array}$ & $\begin{array}{l}\text { GM-20(GC } \dagger) \\
\text { TOKIMEC Co. } \\
\text { Ltd. }\end{array}$ \\
\hline G3 & 9300 & 0.1009 & 0.205 & 2.25 & -9 & $\tau_{1} \fallingdotseq 4$ & $\begin{array}{l}\text { inner } \\
\text { rotor }\end{array}$ & $\begin{array}{l}\text { synchro- } \\
\text { nous } \\
\text { hys- } \\
\text { teresis } \\
\text { motor }\end{array}$ & $\begin{array}{l}\text { JDG-3W } \\
\left.\text { (VG }{ }^{\dagger+}\right) \\
\text { MITSUBISHI } \\
\text { PRECISION } \\
\text { Co. Ltd., } \\
\text { sealed at } 7 \mathrm{kPa} \\
\text { of helium gas } \\
\end{array}$ \\
\hline \multicolumn{10}{|c|}{$\begin{array}{l}*: \text { Calculated amout by the formula proposed by Hayasaka and Takeuchi at the rated speed of right rotation. } \\
* *: \tau_{\gamma} \cdot \tau_{A} \text { and } \tau_{l}: \text { Running times of inertial rotation under vacuum }(10-100 \mathrm{~Pa}) \text {, at atmospheric pressure and the intermediate } \\
\text { pressure }(7 \mathrm{kPa} \text { helium gas }) \\
\dagger: \text { Developed as a gyrocompass }\end{array}$} \\
\hline
\end{tabular}


$170 \phi \times 250 \mathrm{~mm},(2)$ the gyroscope, which is placed in the container, ( 3 ) a light-emitting diode (LED) to be illuminated according to the frequency of the

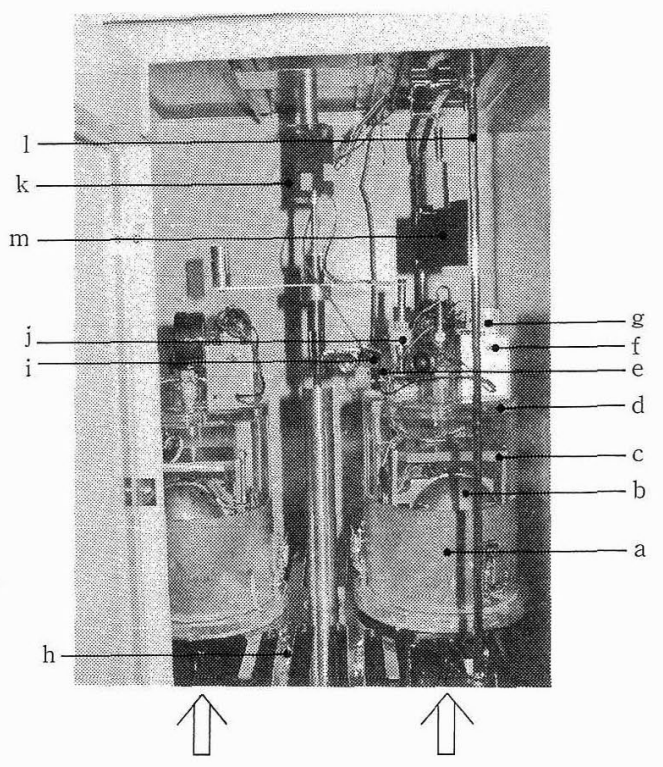

[Reference tare]

[Sample]
a: Cupper foil
b: Gyroscope
c: Holder made of aluminum
d: Vacuum container
e: LED
f: Amplifier for LED
g: Relays

h: Turntable

i: Photosensor

j: Mechanical cutoff switch

k: Stopper for cutoff switch

1: Hanger of balance pan

m: Mirror for twist angle measurement.

Photo. 2 Setting of a sample and the reference tare (RT) : A sample and the RT are on opposite stages in the turntable generated current with the inertial rotation of the rotor in the magnetic fields of the earth and the gyroscope itself, (4) its amplifier with filter and dry batteries set on the container, (5) two relays to switch the connection of the gyroscope from the corresponding power supply to the amplifier, (6) a tap of the mechanical cutoff switch to make the measured object on the weighing system free from the connections for driving the electric motor of the gyroscope or monitoring the rotational speed, and (7) other electric wires.

This system had been proven to be effective in compensating the drift of balance indication as well as in canceling the changes in buoyancy and adsorption on surfaces due to slight changes of environmental conditions ${ }^{6)}$

If there are additional effects due to the difference in the state between the sample gyroscope and the reference one such as magnetic hysteresis, they can be detected by the RT system.

Fig. 1 shows the wiring diagram for the driving or monitoring states. Since the relays in the diagram alternate states by pulsive current, they need no additional current for maintaining the present state.

The balance used ${ }^{6,7)}$ was of the equal-arm type with partially built-in weights, having a capacity of $30 \mathrm{~kg}$ and readability of $0.1 \mathrm{mg}$. Because of the high sensitivity, the transient time of the damped oscillation of the balance indicator was about $60 \mathrm{~s}$. After the transient time passed, the rest points were continuously observed. The balance was also equipped with three special mechanisms which played important roles in

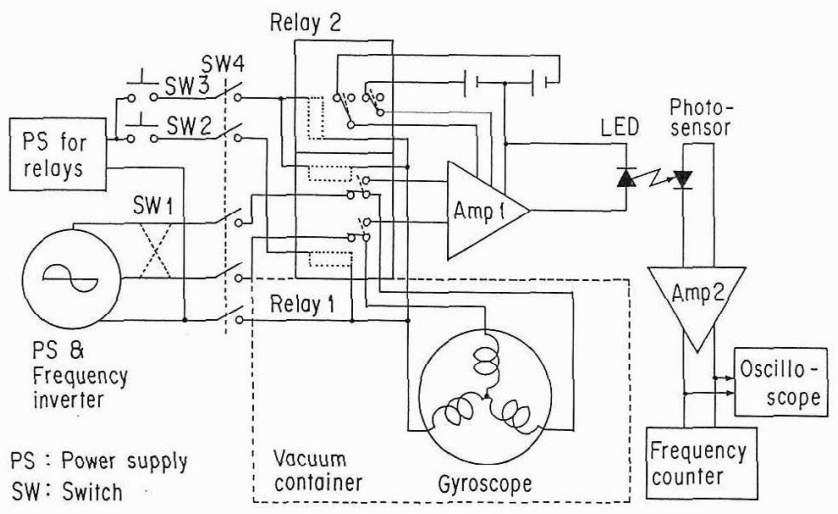

Fig. 1 Wiring diagram for driving a motor of a gyroscope or monitoring the speed of inertial rotation 
the RT system: the first was the load exchange mechanism maintaining the knife edges in contact with the corresponding bearing planes under constant load. The second was the self-adjusting and reproducing mechanism of the vertical alignment of the gravitational center of a measuring body. The third was the hydraulic remote-controlled mechanism to drive the first and second, developed so as not to disturb the state of the measuring objects and the environmental conditions.

\subsection{Monitoring of rotational speed}

As is mentioned above, to realize a non-contact monitoring system of the rotational speed of the rotor in the vacuum container, a mechanical cutoff switch utilizing the up-and-down motion of the turntable of the load exchange mechanism was developed. A small current circuit of $15 \mathrm{~mA}$, including that for the illumination of LED, was introduced to decrease the wear of dry batteries and the generation of Joule heat. The LED was illuminated in accordance with the decrease of the frequency of the generated current of the gyroscope under inertial rotation at the same intensity in spite of the decrease of the voltage. The amplitudes of voltage at the start of inertial rotation of G1, G2 and G3 were about $1 \mathrm{~V}, 2 \mathrm{~V}$ and $60 \mathrm{~V}$, respectively. The frequency of LED illumination was monitored by a photosensor and a frequency counter set apart from the LED, as is shown in Photo. 2.

\subsection{Others}

The twist of the balance pan was easily observed through an optical lever in the direction of the gyroscope rotation because the balance had two pairs of leaf springs as a suspension joint to decrease the shift errors; one was parallel and the other perpendicular to the beam.

Since there were many error factors in the highprecision mass measurement, many other countermeasures were taken: for the adiabatic effect, we used vacuum containers made of acrylic plastics, the lower halves of which were covered with copper foil (see Photo.2) for the conduction between the measured object on the pan and the transporter of the object through the pan, to avoid electrostatic force. For the magnetic effect, holders made of aluminum were used so as to maintain a longer distance between the gyroscope and the transporter of the measured

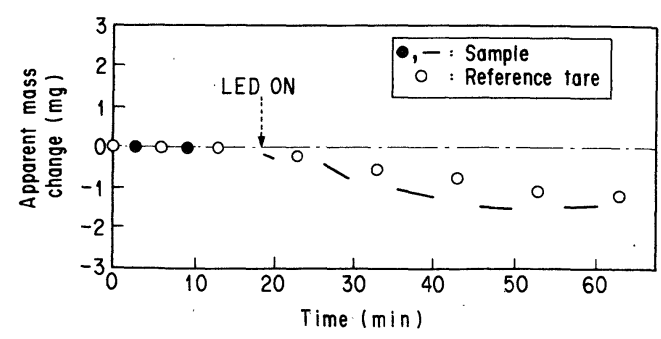

Fig. 2 An example of the stability of apparent mass difference between a sample and the reference tare with respect to the first measured values

object, though G1 and G2 had no residual magnetic field in principle and G3 had the strong sealed case.

\section{Experimental Results}

\section{1 Precision of RT System}

In weighing, "weight reduction" means "mass reduction" because of the high stability of the acceleration of gravity. Hence we use "mass" hereafter as the unit of the measurement. In the first experiment, the stability of the difference in the apparent mass between a sample and the RT was examined. An example is shown in Fig. 2 in the form of the changes from the respective first measured values, though the gyroscope in the sample container was not driven and the LED was illuminated after five exchange measurements to determine the original difference between them. As a slight delay in the difference seemed to depend on the atmospheric pressure change measured by a quartz Bourdon gage (Texas Instrum Inc., model 145-01), measurements were stopped when the pressure was changeable by more than $133 \mathrm{~Pa} / \mathrm{h}$.

After canceling out the common trend between a sample and the RT, we obtained the standard deviation of the RT system of $0.2 \mathrm{mg}$ in 60 minutes, including the leakage of the vacuum container and the wear of dry batteries.

\section{2 Measurement}

Measurements of characteristics of the inertial rotation of gyroscopes under vacuum from 10 to 100 $\mathrm{Pa}$ were made. Inertial rotation speed $\omega$, mass change $\Delta m$ and twist angle $\theta$ of the balance pan with respect to time were observed. An example of such characteristics of G2 is shown in Fig. 3. In the figure, the 


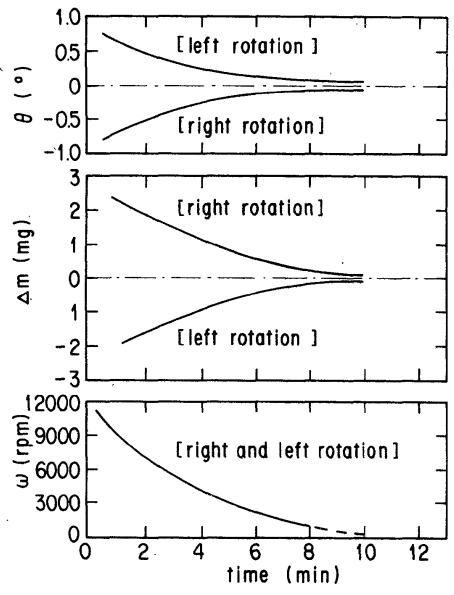

Fig. 3 Measurements for the spinning gyroscope G2 under vacuum.

Inertial rotation speed $\omega$, mass change $\Delta m$ and twist angle $\theta$ of the balance pan with respect to time.

characteristics of $\Delta m$ are shown in the continuous curves, interpolating four series of discrete data.

Fig. 3 also shows that the twist angle $\theta$ decreased symmetrically in accordance with the speed reduction in the direction of rotation, though $\theta$ was not always zero when the rotor stopped because of the hysteresis of the leaf springs.

\section{3 Slight Mass Change due to Rotational Speed}

Fig. 4 shows the apparent mass changes $\Delta m$ of each spinning gyroscope with respect to the rotational speed $\omega$, where the standard deviations at the higher and the much lower region of $\omega$ were about 0.2 and $0.3 \mathrm{mg}$, respectively. No such large and asymmetric effects were observed for any of the gyroscopes tested, as reported by Hayasaka and Takeuchi. The amounts of the observed mass changes for $\mathrm{G} 1$ and $\mathrm{G} 2$ were $1 \%$ and $3 \%$, respectively, of those obtained by the formula proposed by Hayasaka and Takeuchi, although the masses of the rotor of G1 and
G2 were over six times as large as theirs. Therefore, the vertical effect of the spinning gyroscope due to $\omega$ and its product $I \omega$ with the inertia $I$ of the rotor, is neither large nor common.

Irrespective of the large difference in the running time between G1 and G3, the observed mass changes are similar to each other in magnitude. This fact seems to show that the apparent mass changes were affected little by a single factor of the derivative $\omega^{\prime}$.

G3 had magnetic parts in the driving motor, however, and the sealed case decreased the outside magnetic field to a fraction of that of the Earth. The disagreements in the apparent mass change, especially in the right rotation, were variable with the frequency of driving within a range of a few $\mathrm{mg}$; nevertheless, it is evident qualitatively that the vertical effect due to $\omega^{\prime}$ is not significant even in G3 with far shoter running time than the others.

\section{4 Slight Mass Change due to Torque}

Fig. 4 also seems to show that the slight and symmetric mass changes occurred with respect to each $I \omega^{\prime}$ : the change of G2 is about three times as large as that of G1, having equal moment to G1's, while its running time is about one-third as long as G1's, and this is the case for G3. In gyrodynamics, $I \omega^{\prime}$ represents the torque of a spinning gyroscope.

Taking advantage of the flexible-suspension of the pan and the large torque of gyroscopes used in this experiment, we can easily estimate the change in the torque, on the basis of the Hook's law, by measuring the twist angle of the pan, instead of the torque itself.

\subsection{Difference in the Attenuation Constants Between G1 and G2}

The physical differences in the type and environmental conditions between G1 and G2 are expressed by those in the attenuation constants in the higher speed region. The rotational speed $\omega_{i j}(t)$ of gyroscope $G_{i}(i=1$ or 2$)$ and the environmental conditions of
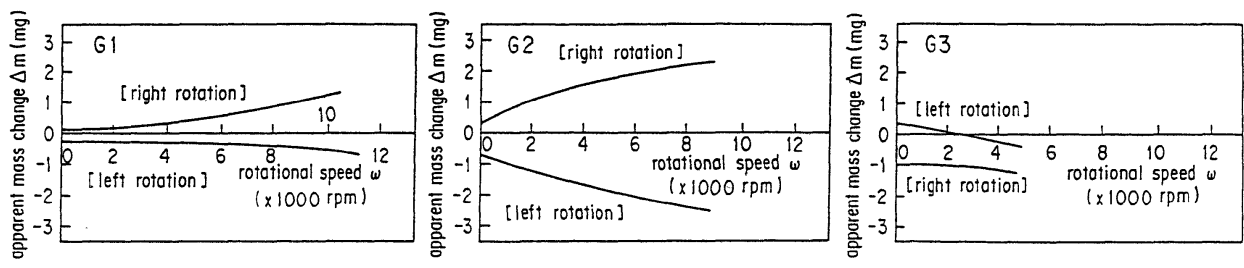

Fig. 4 The observed mass change $\Delta m$ with respect to the rotational speed $\omega$ under vacuum 


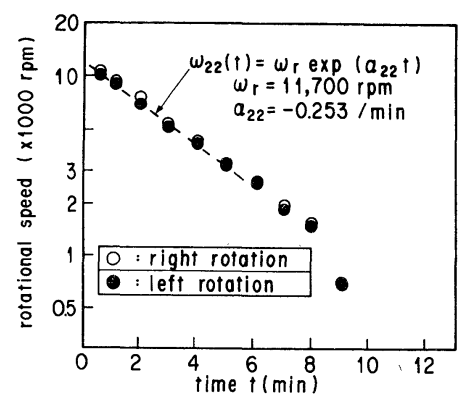

Fig. 5 An example of the intertial rotation speed Gyroscope : G2 $(i=2)$

Condition : under atmospheric conditions $(j=2)$

Table 2 Attenuation constants $\alpha_{i j}$ ' of G1 and G2 under vacuum and at atmospheric pressure

\begin{tabular}{|c|c|c|}
\hline $\begin{array}{l}\text { Condition } \\
\text { Gyroscope }\end{array}$ & $\begin{array}{c}j=1 \\
\text { under vacuum }\end{array}$ & $\begin{array}{l}\quad j=2 \\
\text { at atomospheric } \\
\text { pressure }\end{array}$ \\
\hline $\begin{array}{ll}i=1 & \text { G1 } \\
i=2 & \text { G2 }\end{array}$ & $\begin{array}{l}-0.049 \\
-0.262\end{array}$ & $\begin{array}{l}-0.144 \\
-0.247\end{array}$ \\
\hline
\end{tabular}

the rotor "under vacuum", expressed as $j=1$, or "atmospheric pressure", expressed as $j=2$, are defined. As is shown in Fig. 5, $\omega_{i j}(t)$ was presented as follows in the higher region of $\omega$ of about $3000 \mathrm{rpm}$ or more, irrespective of the direction of rotation:

$$
\omega_{i j}(t)=\omega_{r} \exp \left(\alpha_{i j} \cdot t\right)
$$

where $\omega_{r}$ is the initial speed of G1 and G2 listed as the rated speed in Table 1 , and $\alpha_{i j}$ is an attenuation constant. The $\alpha_{i j}$ 's obtained experimentally are shown in Table 2.

The attenuation constant $\alpha_{21}$ should be smaller than $\alpha_{22}$ due to windage loss; however, this is not the case in the measurement results. This discrepancy reflects the fact that the constants under vacuum and atmosheric conditions at th same time were almost equal each other and that the latter constant was measured posterior to the former, and the running characteristics were changed owing to the frequency of driving.

The ratios of $\alpha_{i j}$ to the minimum $\alpha_{11}$ range from 1 to 5.5. This shows that gyroscopes with large differences in the attenuation constants were examined in this experiment.

In accordance with the general relation

$$
\omega^{\prime}{ }_{i j}(t)=\alpha_{i j} \omega_{i j}(t)
$$

the higher the speed is, the larger is the torque

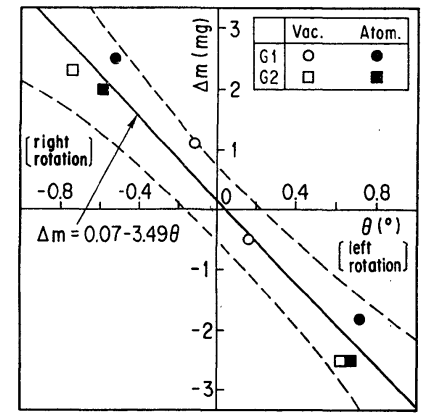

Fig. 6 Relationship between the twist angle $\theta$ of the balance pan and the apparent mass change $\Delta m$ at $9000 \mathrm{rpm}$ of right and left rotations under vacuum (Vac) and atmospheric (Atmo) conditions

$I \cdot \omega^{\prime}{ }_{i j}(t)$ generated, being in direct proportion to the attenuation constant.

The speed $\omega_{\max }$ was taken as $9000 \mathrm{rpm}$ : it is the highest at which the weighing data under the four combinations of $i$ and $j$ were obtained and, under such a condition, the undesirable effect due to heat conductivity from the axis of the gyroscope was negligibly small. Consequently the angular momentum of these gyroscopes at $\omega_{\max }$ is seven times as large as the largest in the previous experiments ${ }^{1)}$.

\section{6 Relationship Between the Twist Angle of the Balance Pan and the Slight Mass Change}

The relationships between the twist angle $\theta$ and the slight mass change $\Delta m$ at $9000 \mathrm{rpm}$ for G1 and G2 are shown in Fig. 6, where the twist of the pan occurred in the direction of the rotation of the rotor.

The relationships of $\mathrm{G} 1$ and G2 shown in the figure are expressed in the same regression equation, although the results of $\mathrm{G1}$ involved largely different cases with respect to the attenuation constant, while those of G2 did not. The slight mass changes for G1 and G2 seemed to occur in terms of torque, but as yet there has been no theory of an interaction between torque and gravity in any cases. Thus we must notice parasitic effects of the measuring system.

As for balances, they are known to have parasitic effects such as shift errors ${ }^{8}$. These are explained by the slight change of the effective arm length due to slight unbalanced forces or momenta newly produced. The direction of the indication change changes accord- 
ing to that of force or momentum. As for the torque of $\mathrm{G} 2$ at $9000 \mathrm{rpm}$, for example, the length change needed to produce $2.5 \mathrm{mg}$ change was $0.05 \mu \mathrm{m}$ under the conditions of the dead weight of $15 \mathrm{~kg}$, the arm length of $250 \mathrm{~mm}$ and the torque of $1.6^{-5} \mathrm{~N} \cdot \mathrm{m}$. The torque was transmitted to the terminal knife edge, through the leaf-spring joint, the cross-knife-edge device and the bearing plane on the knife edge. Such a small length change would occur because shift errors were observed even in this high-presision balance unless the vertical alignment device of the gravity centers between an obfect and the pan was used. The experimental proof of these mechanisms, however, is defficult at the highest level.

Thus we may conclude the apparent mass changes, especially those due to the twist angle at least, were explained mainly by the parasitic effect of the balance used and does not support the existance of a new effect involving $I \omega^{\prime}$.

According to the regression analysis for all the data of G1 and G2, the equation is expressed as follows:

$$
\Delta m=0.07-3.49 \theta(\mathrm{mg})
$$

where the standard deviation $\sigma_{e}$ of residuals is 0.39 mg. The regression line and the confidence limits at the $99 \%$ level are shown in Fig. 6 with straight and dotted lines, respectively.

Resulting from our results, the magnitude of the vertical effect of a spinning gyroscope can be estimated, imagining an ideal balance which is not affected by the torque of spinning gyroscopes at all. This means obtaining the interpolated magnitude of Eq. ( 3 ) at $\theta=0$. For the angular momentum of 0.936 $\mathrm{kg} \cdot \mathrm{m}^{2} / \mathrm{s}$ at $9000 \mathrm{rpm}$, the magnitude under such a condition was $0.07 \mathrm{mg}$, which is less than $0.1 \%$ that of the right rotation obtained by Hayasaka and Takeuchi's formula. It is estimated to be virtually zero with respect to $\sigma_{e}$. The limits with the regression line at the $99 \%$ confidence level, even if a new effect should exist, were neither greater than $+0.7 \mathrm{mg}$ nor less than $-0.5 \mathrm{mg}$, which correspond to the intersection between the confidence limit curves of Eq. ( 3 ) and the line of $\theta=0$.

\section{Discussion}

As for $\mathrm{G} 2$, whose attenuation constants were almost equal to each other within the fluctuation both under vacuum and at atmospheric pressure, no special effects except the parasitic one of the balance were observed even under atmospheric conditions, as long as the attenuation constant was not greatly changed in magnitude and the adiabatic countermeasure was sufficient. The condition of "under vacuum" did not seem to be necessary.

As for the Coriolis force which acts on a spinning body horizontally, the magnitude is $1 \times 10^{-4}$ that of the total weight of the measured object and the pan. It will cause small inclination of the pan hook by $0.3^{\prime}$. The parasitic effect due to such a small inclination was not detected in our weighing system.

\section{Conclusions}

We have examined the vertical effect of spinning gyroscopes with different characteristics using our well-designed and precise experiment. The results are as follows.

1) Results contradicting those of Hayasaka and Takeuchi were obtained, although slight and symmetric effects due to the magnetic parts and the torque of the spinning gyroscopes were observed.

2) The observed symmetric effect of in a few mg was elucidated as a parasitic effect of the balance used due to the torque of spinning gyroscopes, utilizing the change of the attenation constant at atmospheric conditions.

3) For the angular momentum of $0.936 \mathrm{~kg} \cdot \mathrm{m}^{2} / \mathrm{s}$ at $9000 \mathrm{rpm}$, the magnitude of unknown effects is estimated as virtually zero and the existence limits at the $99 \%$ confidence level, if they should exist, are neither greater than $+0.7 \mathrm{mg}$ nor less than $-0.5 \mathrm{mg}$.

This experiment reveals the importance of measurement, and we believe that it is difficult, on account of the instability of the mass of the container itself, to draw a new effect out of such small magnitude against the large dead weight of the container, i.e. about $6 \mathrm{~kg}$.

The authors would like to acknowledge TOKIMEC INC. and MITSUBISHI PRECISION CO. LTD. for lending us their gyroscopes, and Dr. Masanao Morimura and Dr. Kouzo Iizuka for their encouraging advice. We also acknowledge Dr. A. Ohnae for helpful 
discussion.

\section{References}

1) H. Hayasaka and S. Takeuchi : Anomalous Weight Reduction on a Gyroscope's Right Rotations around the Vertical Axis on the Earth, Phy. Rev. Lett., 63, 2701 (1989)

2) J. E. Faller, et al.: Gyroscope-Weighing Experiment with a Null Result, Phy. Rev. Lett., 64, 825 (1990)

3) T. J. Quinn and A. Picard: The Mass of Spinning Rotors : no Dependence on Speed or Sense of Rotation, Nature, 343, 732 (1990)

4) J.M. Nitschke and P.A. Wilmarth: Null Result for the Weight Change of a Spinning Gyroscope, Phy. Rev. Lett. 64, 2115 (1990)

5) A. Imanishi, et al.: Observation against the Weight Reduction of Spinning Gyroscopes, J. Phys. Soc. Jap., 60-4, 1150/1152 (1991)

6) K. Uchikawa, et al. : Production Techniques for Standard Gases by Mass-Measuring Method, Measurement, 1, 54 (1983)

7) Y. Kobayashi : A Study on the Preciser Establishment of Mass Standard (in Japanese), Bull. NRLM, 30, 33 (1981)

8) K. Yamamoto (ed.): Manual for Precise Mass Measurement (in Japanese), 55/56, Japanese Standards Association, Tokyo (1983)

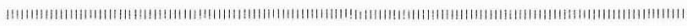

\section{Susumu Ineda (Member)}

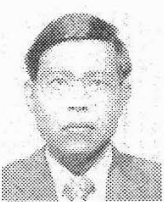

He received B. Eng. from University of Tokyo in 1970, and has been joined the National Research Laboratory of Metrology (NRLM), MITI. $\mathrm{He}$ is a senior researcher of Metrological Standards Section. His research inerests are in surface analytical study of instability of Prototypes of Kilogram and improvements in establishment of mass standards.
Takuma Dor (Member)

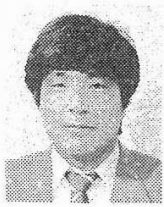

He received M. S. degree in instrumentation from Kobe University in 1986, and joined NRLM in 1987. He belongs to Engineering Measurement Section, working on optical surface profilometry and line-width measurement by optical and electron beams.

\section{Keizaburo U chiкаWa (Member)}

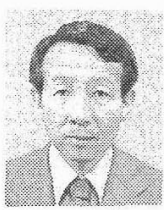

He reccived B. Eng. in electrical engineering from Nippon University in 1960. He joined NRLM and has been the chief of Mechanical Standards Section since 1989. His research interests are in precise establishment of mass standards and relaibility of standard mixture gasses.

\section{Yoshiharu Kurita (Member)}

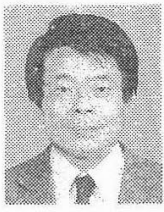

He received M. Ph. from Gakushuin University in 1965, joined NRLM in 1969, and is the Director General.

His research interests are in basic theories of measurement and generation and statistical tests of pseudo-random number.

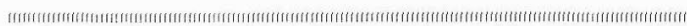

